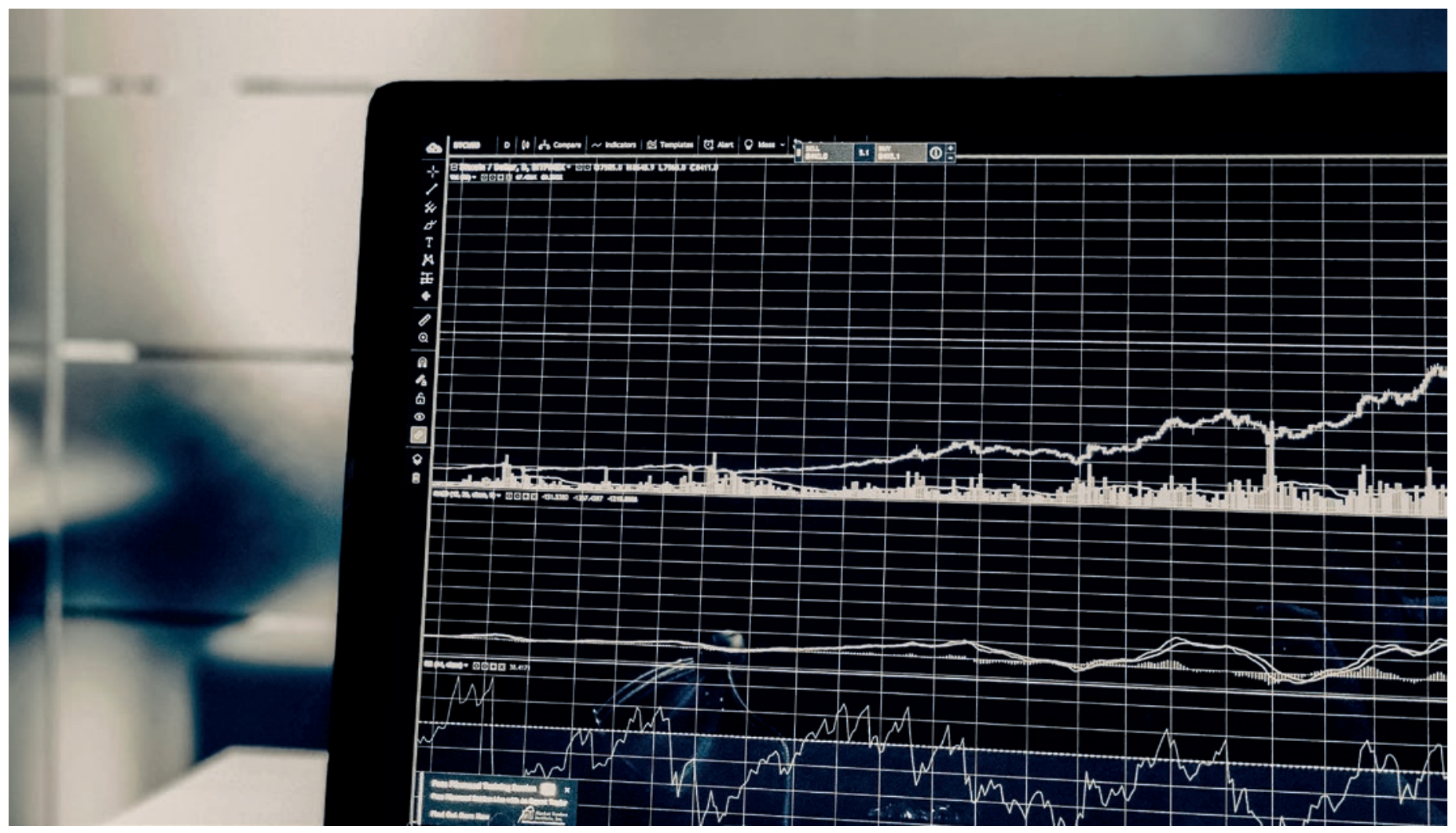

Rechtliche Grundlagen von Evaluationsregistern in der Kranken- und Unfallversicherung

\title{
Evaluationsregister als Allheilmittel bei umstrittenen Leistungen?
}

\section{Marc Wohlwend ${ }^{a}$, Michael Stucki ${ }^{b}$, Philipp Egli $^{\mathrm{c}}$, Simon Wieser ${ }^{\mathrm{d}}$}

${ }^{a}$ RA MLaw, Wissenschaftlicher Mitarbeiter Zentrum für Sozialrecht, ZHAW; ${ }^{b}$ MSc, Wissenschaftlicher Mitarbeiter Winterthurer Institut für Gesundheits-

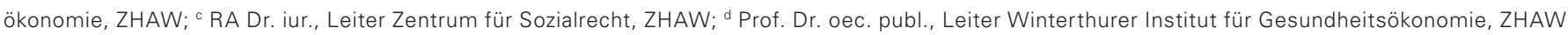

Gesundheitsbezogene Evaluationsregister können wertvolle Daten aus der Alltagsversorgung liefern. Sie werden deshalb regelmässig für gesundheitsökonomische Fragestellungen herangezogen, um zu beurteilen, ob eine Leistung vergütet wird oder nicht. Doch damit sie wirklich zur Schliessung von Evidenzlücken beitragen können, muss einiges beachtet werden.

Sind bei medizinischen Leistungen oder Arzneimitteln die Kriterien der Wirksamkeit, Zweckmässigkeit und Wirtschaftlichkeit umstritten, können Leistungserbringer und/oder Hersteller mit Evaluationsregistern Klarheit schaffen. Diese Register sammeln über einen kurzen Zeitraum von meist wenigen Jahren «real world»-Daten zum Einsatz, zur Wirkung und zu den Kosten der umstrittenen Leistung, um eine evidenzbasierte Vergütungsentscheidung zu ermöglichen. Dank provisorischen Zulassungen haben Patientinnen und Patienten unmittelbaren Zugang zu innovativen medizinischen Leistungen. Allerdings konnten diese Register in der Vergangenheit die Evidenzlücken aus diversen Gründen nicht immer schliessen, und Entscheide über die definitive Vergütung der betroffenen Leistung mussten auf ungenügender Datenbasis gefällt werden. Ist das Evaluationsregister demnach bei umstrittenen Leistungen doch nicht das Allheilmittel? 


\section{Zweck von Evaluationsregistern}

Gesundheitsbezogene Register können vielfältig eingesetzt werden. Sie werden beispielsweise in der klinischen oder epidemiologischen Forschung angewendet oder können der Überwachung von Eingriffen oder von Medizinprodukten dienen. Regelmässig werden gesundheitsbezogene Register auch für gesundheitsökonomische Fragestellungen herangezogen, etwa um die Häufigkeit und die Kosten von einzelnen Behand-

\section{In der Krankenversicherung werden Evalua- tionsregister bei umstrittenen Leistungen in einigen Fällen behördlich angeordnet.}

lungen zu erfassen. Sie können wertvolle Daten aus der Alltagsversorgung liefern und so zur Schliessung vielfältiger Evidenzlücken beitragen.

In der Schweiz und anderen Ländern werden manchmal Evaluationsregister (Register) mit relativ kurzer Laufzeit ins Leben gerufen, um Daten zu sammeln im Hinblick auf eine definitive Aufnahme in den Leistungskatalog der Krankenversicherung oder in den Kreis der Vergütungsempfehlungen der Unfallversicherung.

\section{Weg in den Leistungskatalog}

Damit es eine medizinische Leistung in den Leistungskatalog der sozialen Krankenversicherung schafft, muss sie die Kriterien der Wirksamkeit, Zweckmässigkeit und Wirtschaftlichkeit (WZW-Kriterien) erfüllen, worüber die Bundesverwaltung entscheidet. In der sozialen Unfallversicherung hingegen treffen die Unfallversicherer die nötigen Anordnungen zur zweckmässigen Behandlung der Versicherten (Naturalleistungsprinzip). Die Unfallversicherer vergüten Arzneimittel, pharmazeutische Spezialitäten und Laboranalysen nach den Listen der Krankenversicherung. Die WZW-Kriterien gelten in der Unfallversicherung sinngemäss.

Manchmal ist aber unklar, ob eine Leistung diese Kriterien tatsächlich erfüllt. Sie kann trotzdem unter der Bedingung in den Leistungskatalog (Krankenversicherung) oder unter der Auflage in den Kreis der Vergütungsempfehlungen (Unfallversicherung) aufgenommen werden, dass die Evidenzlücke während einer bestimmten Laufzeit mit einem Register geschlossen wird.

Die aus dem Register gewonnenen Informationen sollen zu einem späteren Zeitpunkt eine fundierte und definitive Entscheidung ermöglichen.

\section{Einsatz von Registern}

Solche Register für Vergütungsentscheidungen werden in drei unterschiedlichen Prozessen eingesetzt:

1 Bei einer provisorischen Aufnahme von Arzneimitteln auf die Spezialitätenliste der Krankenversicherung (SL).

2 Bei einer sogenannten "Coverage with Evidence Development» von ärztlichen Leistungen «in Evaluation", deren Kosten von der Krankenversicherung übernommen werden.

3 Bei einer provisorischen Kostenübernahme von orthopädischen Leistungen durch die Unfallversicherung auf Empfehlung der Medizinaltarif-Kommission UVG (MTK). Hier geht es meist um Implantate. Eine kürzlich erschienene Studie hat die rechtlichen und organisatorischen Herausforderungen im Zusammenhang mit solchen Registern analysiert [1]. Anlass für die Studie war die Tatsache, dass einige der in den letzten Jahren aufgebauten Register die an sie adressierten Fragen nicht beantworten konnten und die Vergütungsentscheidung auf einer ungenügenden Datenbasis gefällt werden musste. Die unfallversicherungsrechtliche Tragweite von Empfehlungen der MTK ist nicht abschliessend geklärt.

\section{Hoheitliche Anordnung}

In der Krankenversicherung werden Evaluationsregister bei umstrittenen ärztlichen Leistungen in einigen Fällen hoheitlich (behördlich) angeordnet. Gesetz und Verordnung sehen diesbezüglich ausdrücklich vor, dass solche Leistungen «nur unter bestimmten Bedingungen» bzw. «nur unter bestimmten Voraussetzungen» übernommen werden [2]. Soweit das Führen eines Evaluationsregisters gemäss Anordnung eine eigentliche Leistungsvoraussetzung (Bedingung) darstellt, ist die Leistung nach der Rechtslehre nur zu entschädigen, wenn das Evaluationsregister auch tatsächlich geführt und gepflegt wird.

\section{Vorgehen bei umstrittenen Leistungen}

In der Unfallversicherung ist der Bundesrat zwar gesetzlich ermächtigt, die Leistungspflicht näher zu umschreiben [3], er hat davon aber bislang nur sehr zurückhaltend Gebrauch gemacht. Namentlich hat der Bundesrat bis heute nicht festgelegt, wer über die Leistungspflicht entscheidet, wenn umstritten ist, ob eine ärztliche Behandlung wirksam sei, und er hat auch nicht bestimmte medizinische Verfahren als «wissenschaftlich anerkannt» oder «wissenschaftlich nicht an- 
erkannt» bezeichnet. Dasselbe gilt sinngemäss für die Erfordernisse der Zweckmässigkeit und der Wirtschaftlichkeit. Umstrittene Arzneimittel vergüten die Unfallversicherer gemäss ausdrücklicher Rechtsgrundlage nach der SL [4]. Bei anderen umstrittenen ärztlichen Leistungen orientieren sich die Unfallversicherer an den Listen der Krankenversicherung, ohne dass dieses

Ob Registerdaten zur Evaluation der Wirksamkeit von medizinischen Behandlungen verwendet werden können, wird kontrovers diskutiert.

Vorgehen gesetzlich ausdrücklich vorgesehen wäre. Bei Arzneimitteln (SL) sind auf Verordnungsstufe insbesondere die Aufnahmebedingungen und die Limitierungen näher geregelt [5]. Das Bundesamt für Gesundheit kann die Aufnahme in die SL allgemein «mit Bedingungen und Auflagen verbinden». Gesetzlich vorgesehen ist insbesondere eine befristete Aufnahme bei Arzneimitteln, deren Wirksamkeit, Zweckmässigkeit oder Wirtschaftlichkeit sich in Abklärung befindet. Ohne gesetzliche Grundlage wäre es unzulässig, ein Arzneimittel, dessen Wirksamkeit (noch) nicht hinreichend belegt ist, mit entsprechender Auflage in die SL aufzunehmen.

Sind beispielsweise orthopädische Leistungen umstritten, empfiehlt die MTK für gewisse Leistungen die Kostenübernahme. Die Empfehlung ist befristet, und während ihrer Laufzeit wird versucht, die Evidenzlücke mit einem Register zu schliessen. Die MTK ist aber nicht damit beauftragt, hoheitlich zu vergütende Leistungen zu bezeichnen oder hoheitliche Massnahmen gegenüber Leistungserbringern zu treffen. Die Empfehlungen der MTK haben aus diesem Grund auch keinen rechtsverbindlichen Charakter.

\section{Verantwortung haben Unfallversicherer}

Die Unfallversicherer haben eine starke Stellung inne: Sie setzen die zweckmässige Leistung gegenüber den Versicherten fest und erteilen den Leistungserbringern (vorgängig zur Behandlung) eine Kostengutsprache. Weil die Unfallversicherer die zweckmässige Leistung festsetzen, sind sie für die Leistung verantwortlich [6]. Die Unfallversicherer können demnach alle notwendigen Anordnungen zur zweckmässigen Behandlung treffen, müssen aber auf die Bedürfnisse der Versicherten und ihrer Angehörigen Rücksicht nehmen (Naturalleistungsprinzip) [7]. Das Verhältnis zwischen Unfallversicherern und Leistungserbringern hingegen ist über Zusammenarbeits- und Tarifverträge, also primär vertraglich, geregelt.
Im Zuge des Naturalleistungsprinzips können die Unfallversicherer Massnahmen anordnen, die in einem engen sachlichen Zusammenhang mit dem jeweiligen Behandlungsfall stehen und verhältnismässig sind. Soweit Register nicht der Beurteilung der Leistungsvoraussetzungen in einem konkreten Behandlungsfall dienen, sondern als Methode der Qualitätsmessung und/oder zu allgemeinen Evaluationszwecken eingesetzt werden, fehlt es möglicherweise am geforderten engen sachlichen Zusammenhang mit dem konkreten Behandlungsfall. Ob nun die Unfallversicherer bei der Umsetzung der Empfehlung der MTK auf dem Führen des Evaluationsregisters beharren können, ist in der Rechtslehre noch ungeklärt.

Hinzu kommt allenfalls ein datenschutzrechtliches Hindernis: Soweit die Registerdaten dem gesetzlichen Zweck dienen, dass (nur) WZW-konforme Leistungen vergütet werden, sind die Unfallversicherer für den Schutz und die Sicherheit der Personendaten verantwortlich [8]. Die Beteiligten eines Evaluationsregisters müssen sich also über die Modalitäten des Registers verständigen.

\section{Mit Registern Wirksamkeit evaluieren?}

Ob Daten aus Registern zur Evaluation der Wirksamkeit von medizinischen Behandlungen verwendet werden können, wird kontrovers diskutiert. Einige Argumente sprechen dagegen. Wichtigster Grund ist das Fehlen einer Kontrollgruppe von Probandinnen und Probanden, die sich von der Interventionsgruppe allein dadurch unterscheidet, dass sie die zu evaluierende Behandlung nicht erhalten hat.

Register weisen im Vergleich zu randomisierten kontrollierten Studien (RCTs), die in der «hierarchy of evidence» der Gesundheitswissenschaften den Goldstandard bilden, trotzdem einige Vorteile auf: Sie erlauben die Beobachtung der Erfolge der Behandlungen unter Alltagsbedingungen. Im Gegensatz zu klinischen Studien beinhalten sie zudem nicht nur Daten eines möglicherweise nicht-repräsentativen Kollektivs. Erfahrungen aus dem Ausland zeigen aber auch, dass bei solchen Registern überproportional häufig Patientinnen und Patienten mit grösseren Komplikationen fehlen.

\section{Register sind in der Lage, Patientinnen und} Patienten über einen deutlich längeren Zeithorizont zu verfolgen als randomisierte Studien.

Register sind - im Gegensatz zu randomisierten Studien - auch in der Lage, Patientinnen und Patienten über einen deutlich längeren Zeithorizont zu verfolgen. Damit können Register in der Versorgungsforschung zur Quantifizierung des sogenannten «Efficacy-Effec- 
tiveness Gap", des Unterschieds der Wirksamkeit unter den kontrollierten Bedingungen der RCTs und der Wirksamkeit unter Alltagsbedingungen, dienen. Aus diesem Grund könnten Register in Zukunft unter anderem auch bei Fragen rund um die personalisierte und die Gender-Medizin zur Anwendung gelangen.

\section{Leistungserbringer und Register}

Dass der Nutzen von Registern für Vergütungsentscheide in der Vergangenheit nicht immer zufriedenstellend war, kann hauptsächlich auf zwei Ursachen zurückgeführt werden. Zum einen wurde der Definition von klaren und umsetzbaren Fragestellungen und

\section{Die Verantwortung für die Registerführung} liegt bei den Ärztinnen und Ärzten, die so mit administrativen Aufgaben belastet werden.

einer sinnvollen Konzeption des Registers nicht immer ausreichend Bedeutung geschenkt. Zum anderen war die Aussagekraft der gewonnenen Evidenz aufgrund der teilweise unzureichenden Vollständigkeit und Vollzähligkeit der Daten begrenzt. Der Bereitschaft der Leistungserbringer zur Datenerfassung kommt daher eine wichtige Rolle zu. Eine vollständige und sorgfältige Registerführung drängt sich unseres Erachtens jedoch auf, weil es sich bezüglich WZW um umstrittene medizinische Leistungen handelt, die der weiteren Evaluation bedürfen.

\section{Teilnahmebereitschaft erhöhen}

Mit der Registerführung ist stets ein bedeutender Zusatzaufwand verbunden, der an ausgebildetes Personal delegiert werden kann. Die Verantwortung bleibt jedoch bei den Ärztinnen und Ärzten, die so nebst der Arbeit mit den Patientinnen und Patienten zusätzlich mit administrativen Aufgaben belastet werden. Es stellt sich daher die Frage, wie die Teilnahmebereitschaft

\section{Das Wichtigste in Kürze}

- Evaluationsregister werden eingesetzt, wenn unklar ist, ob eine medizinische Leistung in den Leistungskatalog der Krankenversicherung bzw. in den Kreis der Vergütungsempfehlungen der Unfallversicherung aufgenommen werden soll.

- Die aus dem Register gewonnenen Informationen sollen eine fundierte und definitive Entscheidung ermöglichen. trotz dieser Ausgangslage erhöht werden kann. Weil auch die Hersteller und die Sozialversicherungen ein besonderes Interesse an einem vollzähligen und vollständigen Register haben, erscheint es zielführend, wenn sie Ärztinnen und Ärzte bei der Registerführung personell und/oder finanziell unterstützen. Es gilt allerdings zu beachten, dass die wissenschaftliche Integrität von Ärztinnen und Ärzten nicht untergraben werden darf und die Daten im Register «unbiased» bleiben müssen.

Bei einem im Anschluss an die eingangs erwähnte Studie durchgeführten Stakeholder-Dialog wurde klar, dass durch einen verstärkten Einbezug der Ärzteschaft bei der Konzeption sowie regelmässige Rückmeldungen von für die Leistungserbringer relevanten Zwischenergebnissen die Motivation zur Teilnahme gesteigert werden kann. Evaluationsregister können jedenfalls - wenn sinnvoll konzipiert und sorgfältig geführt - bei umstrittenen Leistungen heilsam sein.

\section{Danksagung}

Wir danken Prof. Dr. med. Daniel Scheidegger und Susanna Marti Calmell vom Swiss Medical Board für die wertvollen Diskussionen und die hilfreichen Hinweise. Grosser Dank gebührt auch unseren Interviewpartnern, ohne deren umfassendes Praxiswissen wir die Fragestellungen nicht hätten beantworten können. Junia Landtwing danken wir für ihre umfassende Unterstützung im Projekt.

\section{Bildnachweis}

Chris Liverani / Unsplash

\section{Literatur}

1 Wieser S, Landtwing J, Stucki M, Egli P, Wohlwend M. Der Einsatz von Registern bei Vergütungsentscheidungen: Herausforderungen und Handlungsoptionen, 2021 [Internet]. www.swissmedicalboard.ch/fileadmin/public/news/2021/Schlussbericht_SMB_ Register_20210520.pdf. Die Autoren des Artikels haben dieses Gutachten im Auftrag des Swiss Medical Board verfasst.

2 Art. 33 Abs. 3 KVG i.V.m. Art. 33 Bst. a und c KVV respektive Art. 1 Bst. b KLV.

3 Art. 10 Abs. 3 UVG

4 Art. 71 Abs. 2 UVV.

5 Art. 65 und $73 \mathrm{KVV}$

6 Art. 6 Abs. 3 UVG; BGE 136 V 141, E. 4.2.

7 Art. 48 UVG.

8 Art. 16 DSG

\section{L'essentiel en bref}

- Les registres d'évaluation sont utilisés lorsqu'il n'est pas clair si une prestation médicale doit être incluse dans le catalogue des prestations de I'assurance maladie ou parmi les recommandations de remboursement de l'assurance accident.

- Les informations obtenues à partir du registre visent à prendre une décision fondée et définitive. 